\title{
Ensayos a mezclas de barro estabilizadas para el relleno y empañetado de paredes de Bahareque
}

\section{Testing Stabilized Mud Mixtures for Filling and Plastering of Bahareque Walls}

\author{
Henneberg-De León Andrea Mara \\ Universidad del Zulia, Venezuela \\ Facultad de Arquitectura y Diseño \\ Departamento de Construcción y Tecnología en Arquitectura \\ Correo:marahenneberg@yahoo.com
}

\author{
Briceño Daisy \\ Universidad del Zulia, Venezuela \\ Facultad de Arquitectura y Diseño \\ Departamento de Construcción y Tecnología en Arquitectura \\ Correo:ddalizo@yahoo.com
}

Información del artículo: recibido: mayo de 2015, aceptado: julio de 2015

\section{Resumen}

Las edificaciones de bahareque se deterioran mayormente por la vulnerabilidad del barro ante la humedad y por no emplearse mezclas adecuadas en su reparación. Con la finalidad de mejorar las mezclas de barro de los rellenos y los empañetados o revocos de paredes de bahareque, se realizó una investigación cuyos resultados se exponen en este artículo. Esta investigación se basó en el comportamiento de mezclas de barro estabilizadas con cemento o cal, ante los efectos del agua. Para ello, se realizaron una serie de ensayos normados y se diseñó un ensayo de absorción modificado para las mezclas de barro estabilizadas propuestas. Se propusieron mezclas de barro con $10 \%$ de aditivos para los rellenos y hasta $15 \%$ de aditivos para los empañetados. Se le agregó a cada mezcla algunos escombros de bloques de arcilla y cemento para sustituir las piedras que usualmente se emplean en el bahareque del estado de Zulia, Venezuela. Debido a la uniformidad en los resultados de las mezclas con los aditivos de cemento y cal, se concluye que cualquiera de las mezclas propuestas puede utilizarse en una rehabilitación de paredes de bahareque, pero se recomienda la mezcla de barro con $10 \%$ de cemento y bloques de arcilla por ser la que obtuvo los valores más bajos de absorción de agua, es decir, fue la más impermeable.

\section{Descriptores:}

- ensayos

- mezclas de barro estabilizadas

- aditivos de cemento y cal

- relleno

- empañetado 


\begin{abstract}
The buildings of bahareque deteriorate mostly due to the vulnerability of the mud to moisture and the use of not adequate mixtures in its repairs. In order to improve the mud mixtures of the filling and mud cover of bahareque walls, was carried out a research whose results are presented in this article. This research studied the behavior of mud mixtures stabilized with cement and/or lime, to the effects of water. For this purpose a series of standardized tests were performed and it was designed a customized absorption test for the proposed stabilized mud mixtures. Mud mixtures with $10 \%$ of additives for the filling, and up to $15 \%$ of additives for the mud cover were proposed. In the mixtures was also added debris of clay or cement blocks to replace the stones that are usually used in the bahareque of the Zulia state, Venezuela. Due to the uniformity in the results of the mixtures with cement and lime additives, it is concluded that any of the proposed mixtures can be used in a rehabilitation of bahareque walls, but it is recommended the mud mixture with 10 percent of cement and clay blocks, because it had the lowest values of water absorption, that is to say, it is the most waterproof.
\end{abstract}

\section{Introducción}

Dentro de la variedad de técnicas constructivas que utilizan la tierra como material constructivo, se encuentra el bahareque. El bahareque del Estado de Zulia-Venezuela (técnica usada en la región desde el siglo XVII), es el llamado bahareque doble, caracterizado por contar con varas horizontales fijadas a ambos lados de unos horcones de madera que se encuentran alineados. El espacio formado entre las varas se rellena con barro, piedras y otros materiales, para finalmente cubrir todo con barro (revoco o empañetado ${ }^{1}$ ) (figura 1).

La mayoría de edificaciones elaboradas de bahareque que aún persisten, se encuentran deterioradas debido a la vulnerabilidad del barro ante la humedad, asimismo por no utilizar mezclas adecuadas en su reparación (Henneberg de León, 2010).

Con la finalidad de mejorar las mezclas de barro en los rellenos y revocos o empañetados de paredes de bahareque, se realizó una investigación financiada por el Consejo de Desarrollo Científico, Humanístico y Tecno-

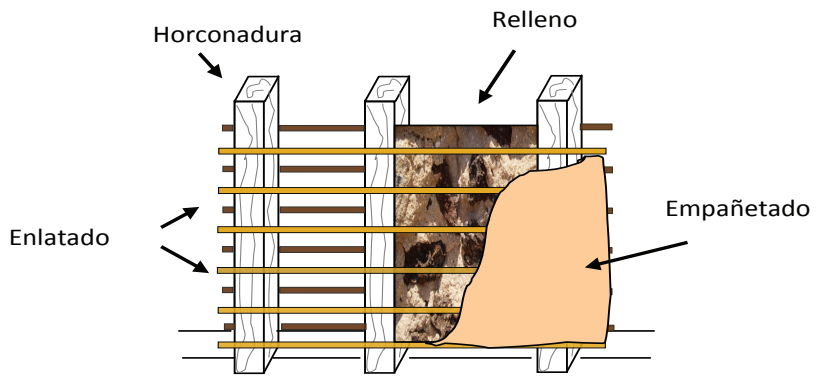

Figura 1. Componentes del bahareque (Henneberg de León, 2012) lógico (CONDES) de la Universidad del Zulia, proyecto VAC-CONDES-CC-0955-08. Esta investigación analizó el comportamiento de mezclas de barro estabilizadas con cemento y cal ante los efectos del agua. Los resultados se muestran en este trabajo.

\section{Desarrollo}

Antecedentes y consideraciones teóricas para el estudio de mezclas

De la gran variedad de aditivos y estabilizantes que se han probado con buenos resultados, se utilizó el cemento y la cal, ya que son materiales del conocimiento popular venezolanos. Además, son materiales que suelen utilizarse para reparar paredes de bahareque en el estado de Zulia, y son de fácil adquisición en el mercado actual.

Debido a que no existen dosificaciones reglamentadas de mezclas de barro con aditivos, se estableció una dosificación basada en varias investigaciones, como las de Díaz et al. (1986), Craterre et al. (1990), Navarro (1995) y Minke (2005). Las proporciones expuestas por estos autores se resumen en la tabla 1.

Las dosificaciones propuestas en el cuadro 1 demuestran que no existe proporción estándar para estabilizar mezclas de barro empleadas en los rellenos y empañetados de un bahareque, pero sí existe cierta correspondencia en los rangos porcentuales para la adición de los estabilizantes de cemento y cal.

En aras de diseñar una mezcla adecuada, este trabajo tomó los siguientes criterios basados en las investigaciones de la tabla 1:

1 Empañetado: Término empleado en Venezuela para denotar un revoco a base de barro. 
Tabla 1. Proporciones de cemento y cal en mezclas de tierra

\begin{tabular}{|c|c|c|c|}
\hline Investigador & País & Estabilizante & Mezcla \\
\hline \multirow[t]{2}{*}{ Díaz et al. 1986} & España & Cemento & $\begin{array}{l}85 \% \text { de tierra y } \\
15 \% \text { de cemento } \\
\text { ó } \\
90 \% \text { de tierra y } \\
10 \% \text { de cemento }\end{array}$ \\
\hline & & Cemento & $\begin{array}{c}\text { Empañetado para muros estabilizados con } \\
\text { cemento: } \\
91 \% \text { de tierra y } \\
9 \% \text { de cemento }\end{array}$ \\
\hline \multirow[t]{2}{*}{ Craterre et al., 1990} & Francia & Cal & $\begin{array}{c}\text { Empañetado para muros estabilizados con } \\
\text { cal o cemento: } \\
91 \text { - } 83 \% \text { de tierra y } \\
9 \text { a } 17 \% \text { de cal }\end{array}$ \\
\hline & & Cemento y cal & $\begin{array}{c}\text { Empañetado para muros estabilizados con } \\
\text { cal o cemento: } \\
89 \text { a } 80 \% \text { de tierra, } \\
9 \text { a } 16 \% \text { de cal y } \\
2 \text { o } 4 \% \text { de cemento }\end{array}$ \\
\hline \multirow[b]{2}{*}{ Navarro, 1995} & & Cemento & $\begin{array}{l}90 \text { a } 95 \% \text { tierra y } \\
5 \text { a } 10 \% \text { cemento }\end{array}$ \\
\hline & Cuba & Cal & $\begin{array}{c}86 \text { a } 97 \% \text { tierra y } \\
3 \text { a } 14 \% \text { cal }\end{array}$ \\
\hline Minke, 2005 & Alemania & $\begin{array}{l}\text { Cemento } \\
\text { Cal }\end{array}$ & $+\operatorname{del} 5 \%$ \\
\hline
\end{tabular}

Fuente: Henneberg de León (2012)

1. Emplear la cantidad de arcilla de acuerdo con la prueba de la cinta (Craterre et al., 1990), a través de una serie de ensayos y errores. Después de realizar varios ensayos con la prueba de la cinta, la mezcla que resistió dicha prueba fue $20 \%$ de arcilla y $80 \%$ de arena.

2. Se resolvió aplicar la media de los porcentajes planteados por las investigaciones analizadas para los aditivos, es decir, se estableció agregar 10\% de aditivo a las mezclas de barro.

3. Para la mezcla del relleno fue preciso realizar dos mezclas: una con $10 \%$ de cemento y la otra con 5\% de cemento y $5 \%$ de cal. El cemento empleado fue Portland 1 color gris, que es común y se encuentra en Venezuela, y la cal seca o aérea, en polvo mayoritariamente cálcica.

3. Considerar para el relleno el uso de un material duro que genere volumen a la mezcla, como ha marcado la tradición de construcción en el estado de
Zulia, en donde generalmente se ha utilizado una mezcla de barro con la llamada "piedra de ojo" (nombre coloquial para una laterita ferruginosa de la zona). Como esta "piedra de ojo" no está disponible en el mercado será reemplazada por escombros de restos de bloques de cemento y de arcilla. Los escombros a utilizar son fragmentos de piezas de ladrillos de cemento y de arcilla cocidos. Los tamaños varian hasta un máximo de $10 \mathrm{~cm}$ y no tienen porosidad.

4. Para el empañetado base se usó la misma mezcla del relleno para garantizar la adherencia entre las mezclas, por ser de la misma composición. Este empañetado base debe aplicarse al mismo tiempo que el relleno.

5. Para el empañetado final o enlucido, se realizaron dos tipos de empañetados: uno con cal y otro con cemento y cal, por lo que se decidió utilizar una de las propuestas de Craterre et al. (1990) referente a los empañetados para muros estabilizados con cal o ce- 
mento (cuadro 1). El empañetado 1 se aplica sobre el relleno con cemento y su mezcla será de $12 \%$ de cal, $3 \%$ de cemento y $85 \%$ de tierra. Para el empañetado 2, la mezcla será de $13 \%$ de cal y $87 \%$ de tierra, y se aplica sobre el relleno con cemento y cal.

6. Se tomó en consideración la propuesta de Craterre et al. (1990) donde se emplean empañetados a la mitad del contenido de arcilla del relleno, para hacerla más liviana que el relleno. Como los empañetados son los verdaderos protectores de la pared, Viñuales (1981) recomienda que estos no deben ser “...tan fuertes que resulten más pesados que el muro y su adherencia se anule en poco tiempo".

Propuesta de mezcla de barro con aditivos de cemento y cal

De acuerdo con los criterios enunciados, en la tabla 2 se resumen las dosificaciones para las mezclas del relleno y el empañetado que se utilizaron en la investigación.

\section{Materiales y métodos}

Ensayos físicos y químicos aplicados a las muestras de mezclas de barro propuestas

En los últimos años las construcciones de tierra han originado gran interés a nivel mundial por emplear materiales naturales y ser consideradas construcciones sostenibles, es por ello que han surgido una serie de estudios y experimentaciones sobre dicha temática en varios países del mundo. Pero las técnicas constructivas que utilizan la tierra como material constructivo no poseen el mismo rigor normativo que el empleado en otros materiales y procesos constructivos.

Existen una serie de normativas para clasificar y estudiar algunas propiedades de los suelos a los cuales se puede recurrir.

Los ensayos físicos realizados en esta investigación fueron: la granulometría, los límites de Atterberg, el ensayo de azul de metileno, el de permeabilidad y el de granulometría por el método del hidrómetro. Estos ensayos se encuentran estandarizados por las normas Fondonorma (Venezuela) y ASTM (EU) y se ejecutaron en los laboratorios del Servicio Autónomo de Ensayos de Materiales (SAEMA) y el de Mecánica de suelos de la Facultad de Ingeniería de la Universidad del Zulia. Los ensayos de permeabilidad y absorción no se realizaron, ya que las mezclas con los aditivos de cemento y cal, quedaban adheridas a los envases de prueba dando como resultado una difícil remoción.

En vista de la importancia del ensayo de absorción, que mide la capacidad de absorber agua (una de las causas que produce las lesiones en las paredes de bahareque), en esta invetigación fue necesario diseñar un ensayo de absorción para las mezclas propuestas, el cual se llamó: ensayo de absorción modificado.

\section{Ensayo de absorción modificado}

Tomando como base el ensayo de absorción (Fondonorma 268:1998) el Laboratorio de Mecánica de Suelos de la Universidad del Zulia junto con la Prof. Henneberg de León diseñaron este ensayo, usando envases de

Tabla 2. Dosificación de mezcla propuesta en este trabajo

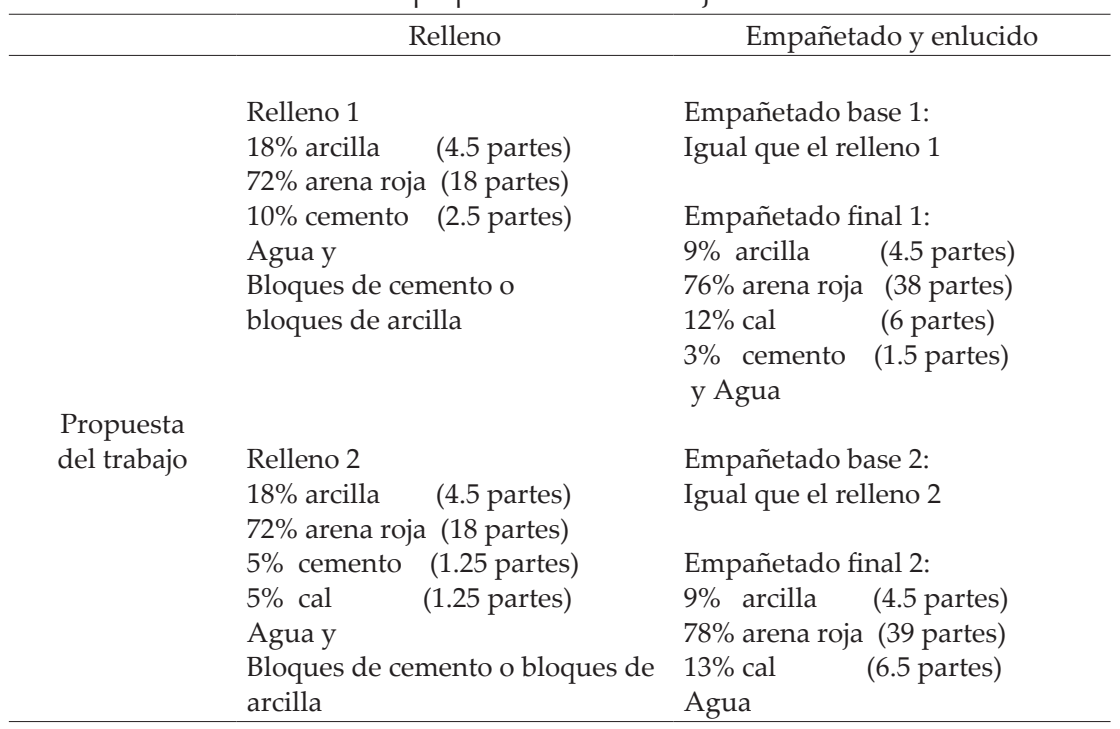

Fuente: Henneberg de León (2010) 
plástico troncocónicos, filtros de papel y recipientes grandes con agua. El proceso fue el siguiente:

1. La muestra de barro con aditivo se colocó en estado plástico dentro de un envase. Los envases utilizados fueron de $13.5 \mathrm{~cm}$ de alto y $8.8 \mathrm{~cm}$ de diámetro.

2. Se dejó secar la muestra aproximadamente un mes a una temperatura ambiente media de $26^{\circ} \mathrm{C}$ y una humedad relativa de $55 \%$.

3. Se removió el fondo del envase de plástico y se hicieron pequeños huecos en el borde superior del envase para que la muestra absorbiera agua por la parte inferior y por la parte superior.

4. Como las muestras tienden a disgregarse por efecto del agua, se colocó un filtro en la parte inferior de los envases (figura 2), situando este envase dentro de otro al que también se le había cortado el fondo (figuras 3 y 4 ).

5. Las muestras en estado seco se pesaron en una balanza electrónica y se registró su valor, es decir, el peso en seco antes del ensayo (figura 5).

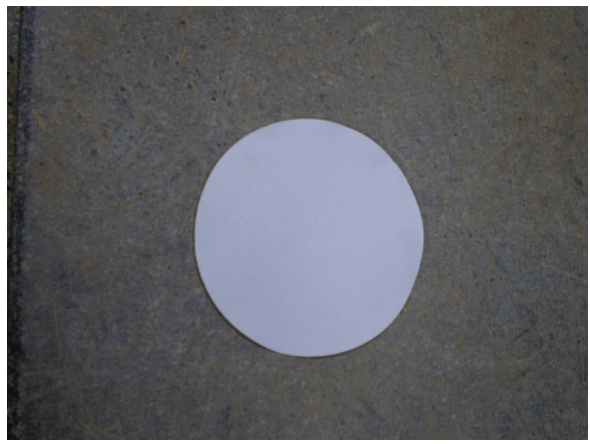

Figura 2. Filtro de papel

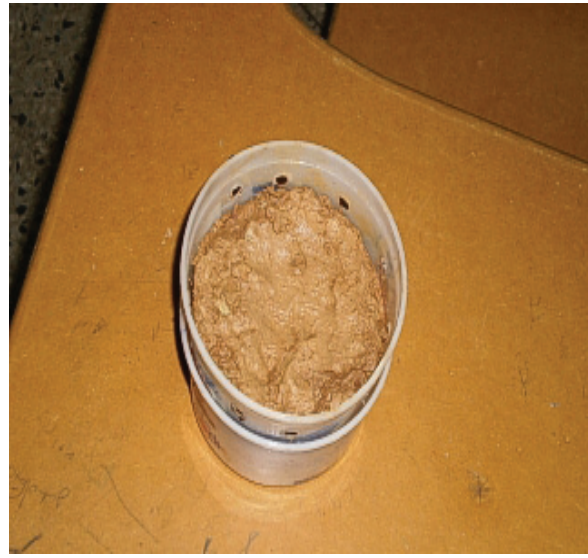

Figura 4. Los dos envases plásticos uno dentro del otro
6. Después se colocaron los envases dobles con las muestras en un recipiente grande con agua, de tal manera que la parte superior de la muestra también quedara bajo agua (figuras 6 y 7 ).

7. Las muestras permanecieron bajo el agua con diferentes lapsos de tiempo: 10, 30 y 60 minutos, 6, 24, 48 horas, 6 y 10 días. Después de cada tiempo se retiraron del agua, se escurrieron y pesaron. Los valores también se registraron.

8. La diferencia entre el peso de la muestra en estado seco y el peso de la muestra a los diferentes intervalos de tiempo suministra el valor de la absorción del agua.

Este ensayo coloca las mezclas en condiciones extremas de humedad, ya que realmente una edificación no permanecerá sumergida en agua, pero indica en caso de una lluvia fuerte y constante cómo se comportaría la mezcla.

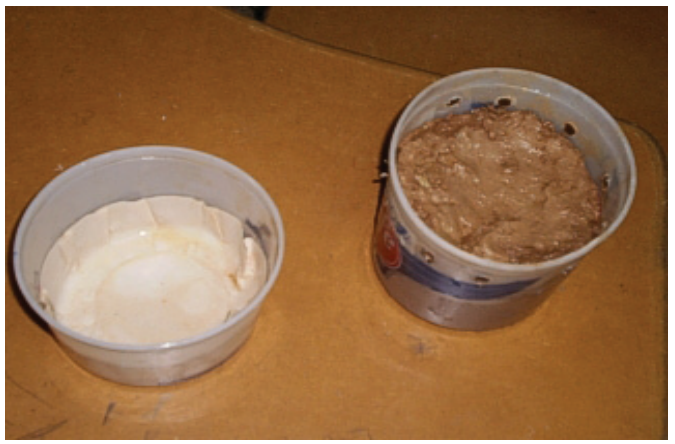

Figura 3. Los dos envases de plástico separados

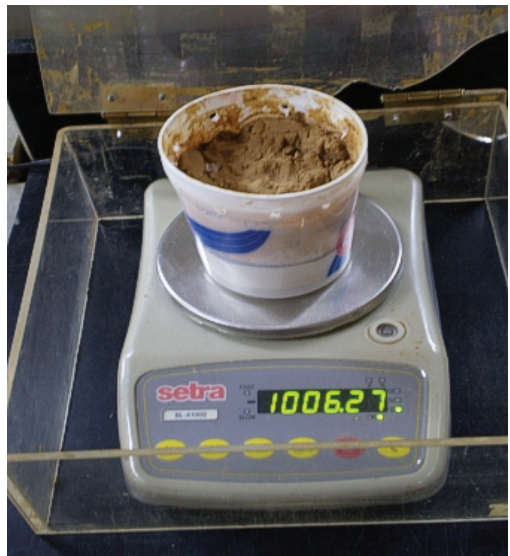

Figura 5. Peso de la muestra antes de sumergirla en agua 


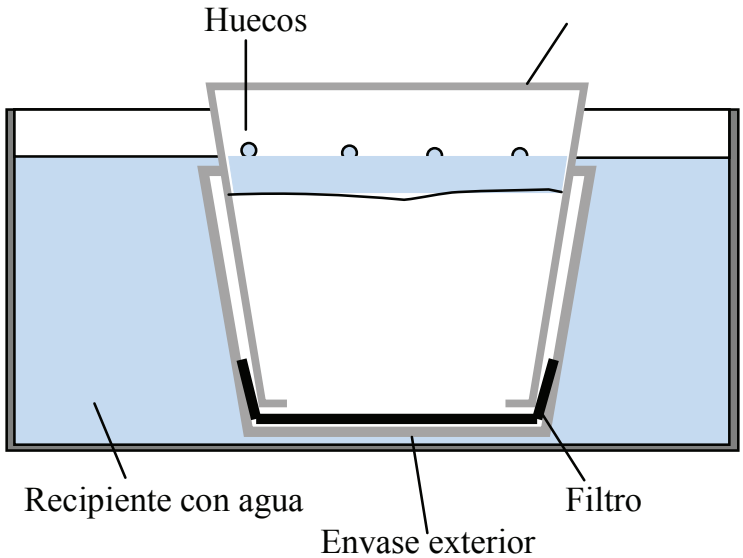

Figura 6. Gráfico del ensayo de absorción modificado Fuente: Henneberg de León (2010)

Con este ensayo es posible conocer de forma aproximada el porcentaje de absorción de agua de las mezclas y, por lo tanto, su grado de impermeabilidad.

Tipos de muestras, nomenclatura y ensayos realizados

Primero se dividieron las muestras en dos grupos de análisis:

Grupo A: Representado por la arcilla y la arena roja a utilizar en las mezclas y

Grupo C: Conformado por las mezclas propuestas en este trabajo:

- Una mezcla de barro.

- Tres mezclas de barro estabilizadas con cemento, con trozos de bloques de arcilla y cemento.

Tres mezclas de barro estabilizadas con cemento y cal con trozos de bloques de arcilla y cemento.

- Una mezcla para el empañetado con cemento y cal.

- Una mezcla para el empañetado con cal.

Se realizaron a su vez, tres muestras $(a, b, c)$ de cada mezcla para poder utilizar su valor promedio. A cada mezcla se le asignó una nomenclatura para diferenciarlas entre sí. La nomenclatura empleada se conformó por tres vocablos: grupo de la muestra, lugar de donde procede la muestra (relleno o empañetado), número general de la muestra.

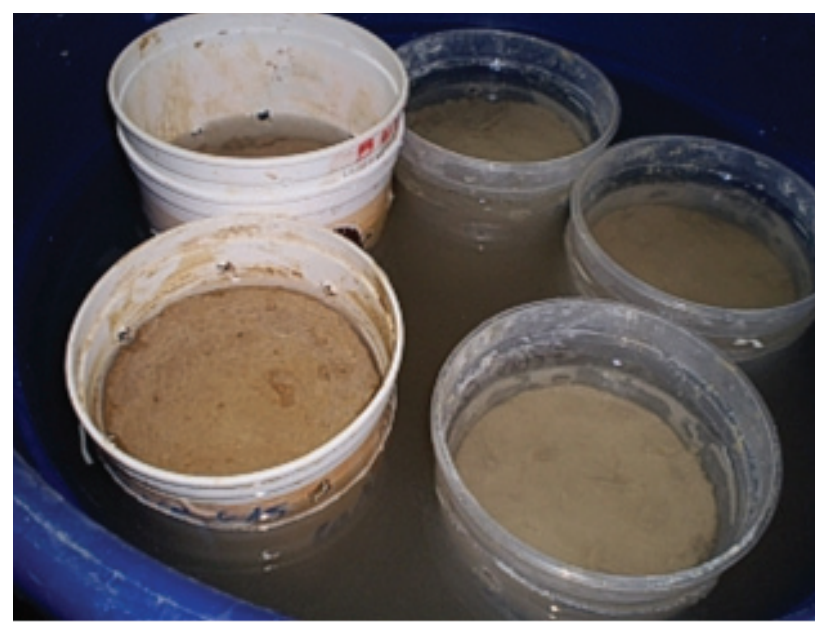

Figura 7. Muestras bajo agua Fuente: Henneberg de León (2010)

A manera de ejemplo, C-rell-01:

$\mathrm{C} \quad$ = identifica al grupo de la muestra

Rell = tipo de muestra, que se utiliza para el relleno

01 = número de la muestra, es decir, primera muestra.

Con el fin de entender la cantidad de muestras, su proveniencia y el tipo de ensayos a los que se sometieron se elaboró la tabla 3.

\section{Grupo A. Muestras de arcilla y arena roja}

Tanto la arcilla como la arena roja utilizadas en la mezcla de barro contaron con varios ensayos.

Fue importante conocer el tipo de arcilla y su plasticidad, por ello se le aplicaron los ensayos de Azul de metileno, límites de Atterberg, granulometría y clasificación de los suelos (tabla 4).

Asimismo se estudió la arena roja a utilizar para saber si tenía algún porcentaje de arcilla que aumentara en la mezcla propuesta.

Por lo tanto, los ensayos aplicados a la arena roja fueron la granulometría por tamizado, la granulometría por hidrómetro y los límites de Atterberg (tabla 4).

\section{Preparación de las muestras}

Muestra de arcilla: La muestra de arcilla se extrajo del saque de Cañada Honda en Maracaibo. La nomenclatura fue: Arcilla con un peso de $500 \mathrm{gr}$.

Muestra de arena roja: De la arena roja se extrajo una cantidad correspondiente a $200 \mathrm{gr}$, cantidad suficiente 
Tabla 3. Tipos de muestras, nomenclaturas y ensayos realizados

\begin{tabular}{|c|c|c|c|c|c|}
\hline Tipo de muestra & Nomenclatura & $\begin{array}{l}\text { Número de } \\
\text { muestras }\end{array}$ & \multicolumn{3}{|c|}{ Ensayos a realizar } \\
\hline Arcilla & A- Arcilla & Then & Azul de metileno & Atterberg & $\begin{array}{l}\text { Granulometría y } \\
\text { clasificación de } \\
\text { suelos }\end{array}$ \\
\hline Arena roja & A - Arena roja & 1 & $\begin{array}{l}\text { Granulometría por } \\
\text { hidrómetro }\end{array}$ & Atterberg & $\begin{array}{l}\text { Granulometría y } \\
\text { clasificación de } \\
\text { suelos }\end{array}$ \\
\hline Mezcla de barro propuesta & C - barro - 07 a,b,c & 4 & $\begin{array}{l}\text { Absorción } \\
\text { modificada }\end{array}$ & & Permeabilidad \\
\hline $\begin{array}{l}\text { Mezcla de relleno propuesta } \\
(\text { barro }+ \text { cem }+ \text { bloq arcil) }\end{array}$ & C - rell - $08 \mathrm{a}, \mathrm{b}, \mathrm{c}$ & 3 & $\begin{array}{l}\text { Absorción } \\
\text { modificada }\end{array}$ & & \\
\hline $\begin{array}{l}\text { Mezcla de relleno propuesta } \\
\text { (barro }+ \text { cem + bloq arcil + } \\
\text { bloq cem) }\end{array}$ & C - rell - $09 a, b, c$ & 3 & $\begin{array}{l}\text { Absorción } \\
\text { modificada }\end{array}$ & & \\
\hline $\begin{array}{l}\text { Mezcla de relleno propuesta } \\
\text { (barro + cem + bloq cem) }\end{array}$ & $C$ - rell - $10 a, b, c$ & 3 & $\begin{array}{l}\text { Absorción } \\
\text { modificada }\end{array}$ & & \\
\hline $\begin{array}{l}\text { Mezcla de relleno propuesta } \\
(\text { barro }+ \text { cal }+ \text { cem + bloq arcil) }\end{array}$ & $C$ - rell - $11 a, b, c$ & 3 & $\begin{array}{l}\text { Absorción } \\
\text { modificada }\end{array}$ & & \\
\hline $\begin{array}{l}\text { Mezcla de relleno propuesta } \\
(\text { barro }+ \text { cal }+ \text { cem }+ \text { bloq arcil } \\
+ \text { bloq cem })\end{array}$ & $C$ - rell - $12 a, b, c$ & 3 & $\begin{array}{l}\text { Absorción } \\
\text { modificada }\end{array}$ & & \\
\hline $\begin{array}{l}\text { Mezcla de relleno propuesta } \\
(\text { barro }+ \text { cal }+ \text { cem + bloq cem })\end{array}$ & $C$ - rell - $13 a, b, c$ & 3 & $\begin{array}{l}\text { Absorción } \\
\text { modificada }\end{array}$ & & \\
\hline $\begin{array}{l}\text { Mezcla de empañetado } \\
\text { propuesta } \\
(\text { barro }+ \text { cem }+ \text { cal })\end{array}$ & C - emp - 14 a,b,c & 3 & $\begin{array}{l}\text { Absorción } \\
\text { modificada }\end{array}$ & & \\
\hline $\begin{array}{l}\text { Mezcla de empañetado } \\
\text { propuesta } \\
\text { (barro + cal) }\end{array}$ & C - emp - $15 a, b, c$ & 3 & $\begin{array}{l}\text { Absorción } \\
\text { modificada }\end{array}$ & & \\
\hline
\end{tabular}

Fuente: Henneberg de León (2012)

Tabla 4. Ensayos realizados a la arcilla y a la arena

\begin{tabular}{|c|c|c|c|c|}
\hline Ensayo & Razón del ensayo & $\begin{array}{l}\text { Laboratorio donde se } \\
\text { realizó el ensayo }\end{array}$ & $\begin{array}{c}\text { Resultado } \\
\text { Muestra arcilla }\end{array}$ & $\begin{array}{c}\text { Resultado } \\
\text { Muestra arena roja }\end{array}$ \\
\hline $\begin{array}{l}\text { Granulometría por } \\
\text { tamizado }\end{array}$ & $\begin{array}{l}\text { Determina el tipo de } \\
\text { suelo }\end{array}$ & $\begin{array}{l}\text { SAEMA } \\
\text { De acuerdo con las } \\
\text { normas IE-T-401 y } \\
\text { COVENIN 255- } 2006\end{array}$ & $\begin{array}{l}\text { La muestra es una } \\
\text { ARCILLA }\end{array}$ & $\begin{array}{l}\text { Arena con un porcentaje } \\
\text { de arcilla }\end{array}$ \\
\hline $\begin{array}{l}\text { Granulometría } \\
\text { por hidrómetro }\end{array}$ & $\begin{array}{l}\text { Establece la } \\
\text { presencia o no de } \\
\text { arcilla en la muestra }\end{array}$ & $\begin{array}{l}\text { Laboratorio de Mecánica } \\
\text { de suelos de la Facultad } \\
\text { de Ingeniería de la } \\
\text { Universidad del Zulia }\end{array}$ & & $\begin{array}{l}\text { Existe } 2.63 \% \text { de arcilla } \\
\text { en la muestra de arena } \\
\text { roja }\end{array}$ \\
\hline $\begin{array}{l}\text { Límites de } \\
\text { consistencia o de } \\
\text { Atterberg }\end{array}$ & $\begin{array}{l}\text { Establece la } \\
\text { plasticidad de los } \\
\text { suelos }\end{array}$ & $\begin{array}{l}\text { SAEMA } \\
\text { De acuerdo con las } \\
\text { normas IE-T.410 y } \\
\text { COVENIN 3548-99 }\end{array}$ & $\begin{array}{l}\text { Suelo con alta } \\
\text { plasticidad }\end{array}$ & $\begin{array}{l}\text { Es un suelo con } \\
\text { baja plasticidad } \\
\text { correspondiente a las } \\
\text { arenas }\end{array}$ \\
\hline Azul de metileno & $\begin{array}{l}\text { Determina el tipo de } \\
\text { arcilla }\end{array}$ & SAEMA & Es una vermiculita & \\
\hline $\begin{array}{l}\text { Clasificación de los } \\
\text { suelos }\end{array}$ & $\begin{array}{l}\text { Clasifica y } \\
\text { determina el tipo de } \\
\text { suelo }\end{array}$ & SAEMA & $\begin{array}{l}\text { De acuerdo con HRB es } \\
\text { un A-7-6, es decir, suelos } \\
\text { arcillosos } \\
\text { De acuerdo con SUCS } \\
\text { (Sistema unificado de } \\
\text { clasificación de suelos) } \\
\text { es un CH = arcillas } \\
\text { inorgánicas de alta } \\
\text { plasticidad }\end{array}$ & $\begin{array}{l}\text { De acuerdo con el } \\
\text { Sistema unificado de } \\
\text { clasificación de suelos } \\
\text { es una SM - SC = arena } \\
\text { limo-arcillosa }\end{array}$ \\
\hline
\end{tabular}

Fuente: Henneberg de León (2012) 
para realizar los diferentes ensayos. La nomenclatura fue: Arena roja.

Grupo C. Muestras de la mezcla de barro propuesta y de las diferentes mezclas de barro con aditivos de cemento, cal y relleno de bloques de cemento y bloques de arcilla

A la mezcla de barro propuesta se le aplicaron los ensayos de permeabilidad y de absorción modificada para conocer su capacidad de absorción ante la exposición al agua.

De las mezclas propuestas eran conocidas las proporciones de los materiales, la composición química y la granulometría, por lo que solo se ensayó la absorción de agua.

\section{Preparación de las muestras}

Primero se realizó la mezcla de barro propuesta, donde se elaboraron tres muestras para usar un promedio de los tres resultados. La nomenclatura empleada fue de: C-barro-7 a, C-barro 7 b y C-barro-7 c.

Para las mezclas del relleno se deseaba ensayar los dos tipos de mezclas con la adición de los aditivos propuestos: barro con cemento y barro con cemento y cal, junto a la parte dura del relleno, es decir, los escombros de bloques. Para conocer cuál de las mezclas absorben más o menos humedad se procedió a la elaboración de muestras con los bloques por separado y unidos, haciendo de cada mezcla tres muestras.

De ello resultaron las siguientes mezclas y sus respectivas nomenclaturas:

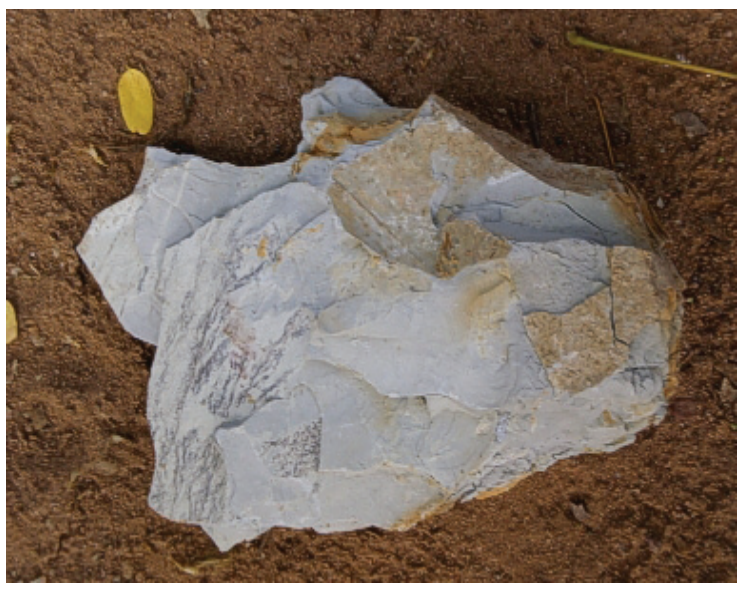

Figura 8. Vermiculita en estado natural (Henneberg de León, 2010)
- Barro con cemento y bloque de arcilla: C-rell-08a, Crell-08b y C-rell-08c

- Barro con cemento y bloque de arcilla y bloque de cemento: C-rell-09a, C-rell-09b y C -rell-09c

- Barro con cemento y bloque de cemento: C-rell-10a, C-rell-10b y C-rell-10c

- Barro con cemento y cal y bloque de arcilla: C-rell11a, C-rell-11 y C-rell-11c

- Barro con cemento y cal y bloque de arcilla y bloque de cemento: C-rell-12a, C-rell-12b y C-rell-12c

- Barro con cemento y cal y bloque de cemento: Crell-13a, C-rell-13b y C-rell-13c

Por último, se hicieron las mezclas de los dos tipos de empañetado propuesto. Haciendo también tres muestras de cada tipo de mezcla. Sus respectivas nomenclaturas fueron las siguientes:

- Barro con cemento: C-emp-14 a, C-emp-14 b y Cemp-14 c

- Barro con cemento y cal: C-emp-15 a, C-emp-15 b y C-emp-15 c-

\section{Resultados}

Resultados de los ensayos aplicados a la arcilla y a la arena roja- Grupo A

Los ensayos aplicados a la muestra de arcilla establecen que es una vermiculita de alta plasticidad. La vermiculita está compuesta por silicatos de aluminio, magnesio y de hierro. "La fórmula estructural aproximada de la vermiculita es: $\mathrm{Mg}_{3}\left(\mathrm{Al}, \mathrm{Si}_{4} \mathrm{O}_{10}(\mathrm{OH})_{2} \mathrm{Mg}_{0.35}-4.5 \mathrm{H}_{2} \mathrm{O}^{\prime \prime}\right.$ (Avgustinik, 1983, p.56). La parte de $\mathrm{Mg}_{0.35}$ puede sustituirse por $\mathrm{Fe}^{3}+, \mathrm{Fe}^{2}+, \mathrm{Al}$, Ca y Cu. Su forma natural es de una estructura laminar de color pardo o gris (figura 8). Es inodora y no tóxica.

En cuanto a la arena, la muestra es una arena-limoarcillosa, con $2.63 \%$ de arcilla, porcentaje que debe añadirse a la dosificación porcentual de la mezcla de barro propuesta.

Resultados de la mezcla de barro propuesta. Ensayo: Permeabilidad- Grupo C

Este ensayo se realizó en el Laboratorio de Mecánica de suelos de la Universidad del Zulia. La muestra se sometió al proceso de saturación para posteriormente realizar el ensayo de permeabilidad.

La mezcla C-barro-7 estuvo en agua por aproximadamente 70 días y no llegó a saturarse $100 \%$. En vista de que solo había un equipo disponible, la muestra se tuvo 
que retirar sin lograr finalizar el ensayo de permeabilidad. No se lograron resultados, pero el hecho de que la muestra de barro no consiguiera la saturación de agua a $100 \%$, indica que la muestra de barro tiene un alto grado de impermeabilidad.

Resultado de la mezcla de barro propuesta. Ensayo: Absorción modificado- Grupo C

Este ensayo se realizó en el Laboratorio de Mecánica de Suelos de la Universidad del Zulia. A la muestra de barro propuesta C-barro-7 se le aplicó el ensayo de absorción modificado para que sirviera como muestra patrón y se comparara con las mezclas de barro con aditivos.

La mezcla de barro propuesta aumentó su peso en 10 minutos a $13.97 \%$ aumentando gradualmente hasta llegar a $17.70 \%$ en 10 días (figura 9)

Resultado de las diferentes mezclas de barro con aditivos de cemento, cal y relleno de bloques de cemento y bloques de arcilla. Ensayo: Absorción modificado- Grupo C

Este ensayo se hizo en el Laboratorio de Mecánica de Suelos de la Universidad del Zulia y se condujo por la Dra. Henneberg de León. Con este ensayo se manifestó de forma aproximada la capacidad de absorción de agua en las mezclas, calificándolas de más o menos impermeables.

Los valores observados en las diferentes pesadas con los tiempos estipulados para tal fin, se registraron en una tabla. A cada peso se le calculó el porcentaje de aumento de peso respecto al peso inicial en seco de la muestra. Posteriormente se promediaron los valores de las tres muestras iguales por cada mezcla, para conocer el valor de aumento o disminución de peso (producto de la disgregación del material) que experimentó cada mezcla.

De las diferentes mezclas con aditivos para el relleno se observó que casi todas se comportaron de forma similar, contaron con aumentos y disminuciones. La característica más sobresaliente fue que después de los primeros 10 minutos los pesos no aumentaron significativamente, manteniéndose en un orden de $2 \%$ entre el peso a los 10 minutos en agua y el peso de 10 días en agua (figura 9).

Las mezclas para el empañetado sí mostraron diferencias entre ellas. La mezcla con barro, cal y cemento mostró un aumento entre $12.10 \%$ hasta $14.00 \%$ y una disminución de peso, mientras que la mezcla de barro con cal aumentó entre $17.20 \%$ y $19.20 \%$ y tuvo varias disminuciones de peso (figura 10).
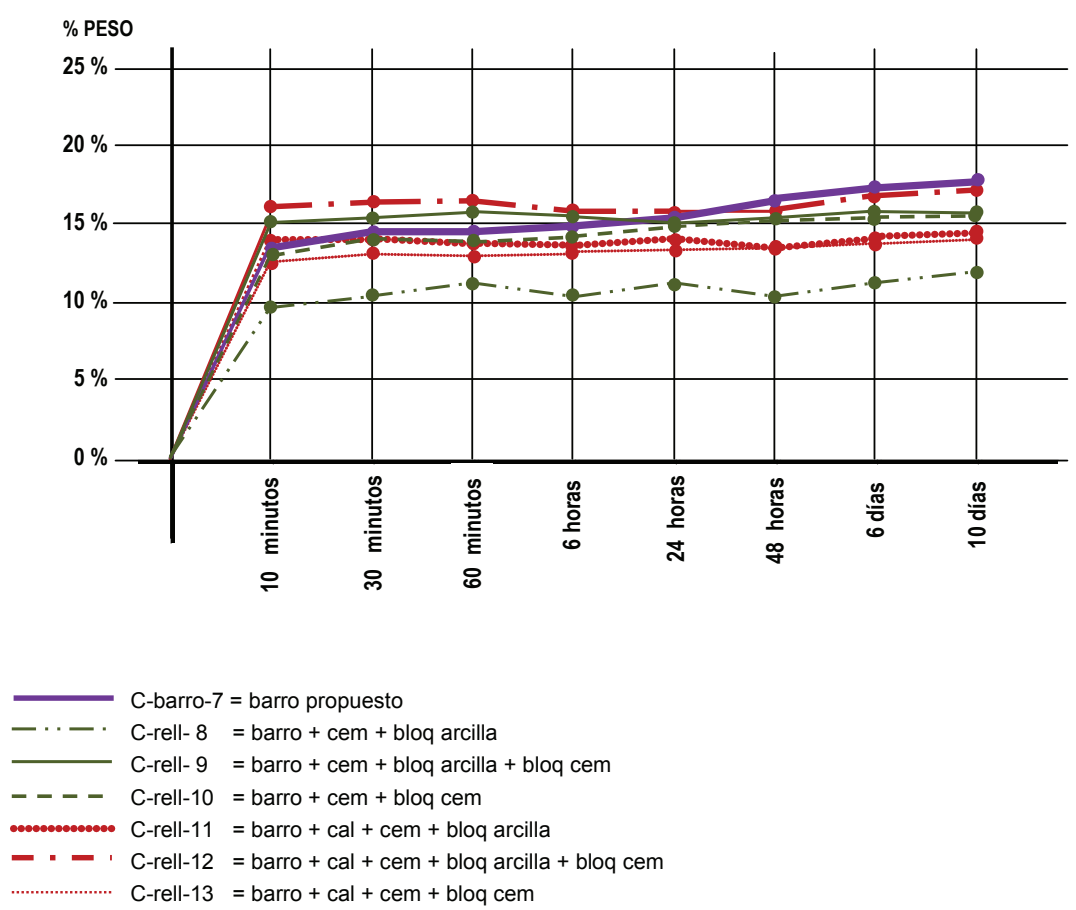

Figura 9. Gráfico con promedios de \% del ensayo de absorción modificado en mezclas de relleno

Fuente: Henneberg de León (2012) 


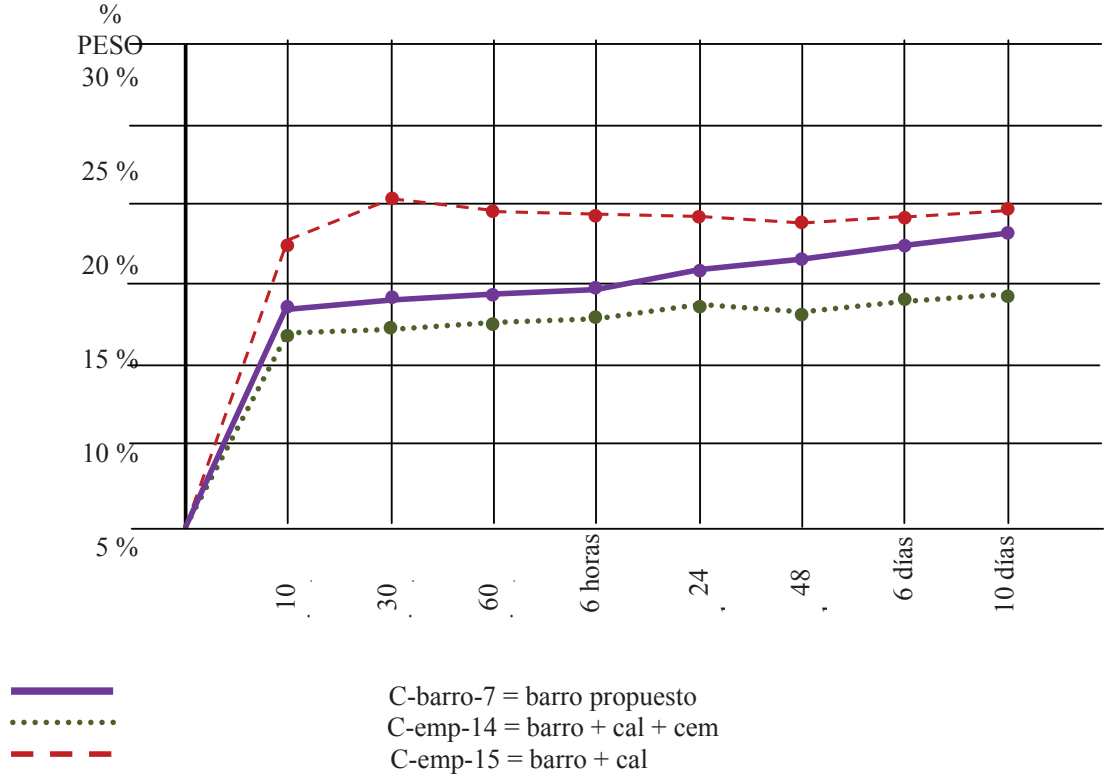

Figura 10. Gráfico de promedios de \% del ensayo de absorción modificado en mezclas de empañetado. Fuente: Henneberg de León (2012)

\section{Discusión de los resultados de los ensayos}

Los porcentajes de aumento en la mezcla de barro propuesta, NO es un porcentaje de aumento significativo, lo que demuestra que la mezcla de barro tiene un grado medio de impermeabilidad. No existieron disminuciones de peso, por lo que también se puede decir que no se disgrega fácilmente en el agua.

Los resultados de las diversas mezclas con aditivos para el relleno demuestran que las mezclas tienen una mayor capacidad de absorción en los primeros minutos de permanecer sumergidas $y$, después de ese tiempo su capacidad de absorción disminuye drásticamente, posiblemente porque los aditivos de la mezcla reducen esta capacidad disminuyendo los espacios vacíos dentro de la mezcla.

En la figura 9 se reflejan los porcentajes de peso calculados para las diversas mezclas, así como la propuesta de barro para poder hacer comparaciones. Se tiene que:

- La mezcla de barro propuesta siempre absorbió agua de forma ascendente hasta llegar a $17.7 \%$ en 10 días.

- Las demás mezclas, aunque absorbieron más o menos cantidad que la mezcla de barro propuesta a lo largo del tiempo, el valor del porcentaje a los 10 días está por debajo de $17.70 \%$. Esto indica que las mezclas con aditivos y escombros son más impermeables que la mezcla de barro propuesta o que van disminuyendo su capacidad de absorción hasta llegar a los 10 días. Es decir, tienen mayor grado de impermeabili- dad comparándolo con la impermeabilidad de la muestra de barro sin aditivos.

- Casi todas las mezclas con aditivos arrojaron porcentajes de aumento de peso entre $9.97 \%$ y $17.40 \%$, respecto al peso inicial.

- La mezcla que resultó más impermeable fue la Crell-08, hecha con barro, cemento y bloque de arci1la. Luego en orden descendente, la C-rell-13, la C-rell-11, la C-rell-10, la C-rell-9 y la C-rell-12.

Respecto a las mezclas para el empañetado se puede establecer lo siguiente:

- El empañetado con cemento y cal es más impermeable y se disgrega mucho menos que el empañetado con solo cal.

- De acuerdo con el gráfico de curvas (figura 10) elaborado con los valores del empañetado y del barro propuesto (como patrón), el empañetado con cemento y cal es más impermeable que el barro, ya que los valores del empañetado a base de cal son mayores y se encuentran por encima, incluso, de la curva del barro propuesto.

\section{Conclusiones}

a) En vista del contenido de arcilla de $2.63 \%$ en la arena roja, la dosificación real de la mezcla de barro propuesta fue de $22.63 \%$ arcilla y $77.37 \%$ arena roja.

La dosificación real de la mezcla propuesta es la que se muestra en la tabla 5. 
Tabla 5. Dosificación real de mezcla propuesta en este trabajo

\begin{tabular}{|c|c|c|}
\hline & Relleno & Empañetado y enlucido \\
\hline & Relleno 1 & Empañetado base 1: Igual que el relleno 1 \\
\hline \multirow{3}{*}{$\begin{array}{l}\text { Propuesta real } \\
\text { del trabajo }\end{array}$} & $\begin{array}{c}20.63 \% \text { arcilla ( } 5.2 \text { partes), } \\
\text { 69.37\% arena roja (17.3 partes), } 10 \% \\
\text { cemento ( } 2.5 \text { partes), Agua, Bloques } \\
\text { de cemento y bloques de arcilla }\end{array}$ & $\begin{array}{c}\text { Empañetado final 1: } \\
11.63 \% \text { arcilla (5.8 partes), } \\
73.37 \% \text { arena roja (36.7 partes), } 12 \% \text { cal (6 } \\
\text { partes), } \\
3 \% \text { cemento ( } 1.5 \text { partes) y Agua }\end{array}$ \\
\hline & $\begin{array}{c}\text { Relleno } 2 \\
\text { 20.63\% arcilla ( } 5.2 \text { partes), }\end{array}$ & Empañetado base 2: Igual que el relleno 2 \\
\hline & $\begin{array}{c}69.37 \% \text { arena roja (17.3 partes), } 5 \% \\
\text { cemento (1.25 partes), } 5 \% \text { cal }(1.25 \\
\text { partes), } \\
\text { Agua, Bloques de cemento y } \\
\text { bloques de arcilla }\end{array}$ & $\begin{array}{c}\text { Empañetado final 2: } \\
11.63 \% \text { arcilla ( } 5.8 \text { partes), } \\
75.37 \% \text { arena roja (37.7partes), } 13 \% \text { cal } \\
\text { (6.5 partes), Agua }\end{array}$ \\
\hline
\end{tabular}

Fuente: Henneberg de León (2010)

b) Sobre la mezcla propuesta para el relleno usando como aditivo el cemento: De acuerdo con las investigaciones de diferentes autores mundiales y nacionales, la adición de cemento entre 5 a 15\% permite impermeabilizar el barro. En este trabajo se utilizó 10\% de cemento como aditivo a la mezcla de barro, proporción que logra una cierta impermeabilización dentro del marco de lo sostenible.

c) Sobre la mezcla propuesta para el relleno usando como aditivo el cemento y la cal: Para la adición de cemento y cal se mantuvo la proporción de 10\%, dividiéndolo en $5 \%$ de cal y $5 \%$ de cemento. Los resultados de esta mezcla con los diferentes rellenos de bloques también se manifestaron por debajo de los valores de la mezcla de barro sin aditivos, es decir, que también impermeabilizan la mezcla. Esta mezcla se puede utilizar cuando se desee disminuir el porcentaje de cemento.

d) Sobre la mezcla propuesta para el empañetado usando como aditivo el cemento y la cal: Fundamentado en otras investigaciones se decidió adicionar cemento con una proporción de $3 \%$ y cal con proporción de $12 \%$. Esta mezcla mostró valores por debajo de los valores del barro sin aditivo, concluyendo que logró cierta impermeabilización respecto al barro. Se recomienda utilizar esta mezcla para los empañetados.

e) Sobre la mezcla propuesta para el empañetado usando como aditivo la cal: En esta mezcla se resolvió adicionar $13 \%$ de cal, basado en las propuestas de otras investigaciones. Esta mezcla obtuvo valores por encima de la mezcla de barro sin aditivos, es decir, que no la impermeabiliza, más bien aumenta su permeabilidad. Por lo que NO se recomienda.

f) Los porcentajes de aumento debido a la absorción de agua de todas las mezclas propuestas se mantienen en un rango entre $9.97 \%$ y $22.10 \%$, porcentajes relativamente bajos en cuanto a la impermeabilidad. Esto indica que solo existe cierta impermeabilidad.

Debido a la relativa homogeneidad de los resultados de las mezclas con aditivos se concluye que cualquiera de las mezclas propuestas con aditivos se puede emplear en una rehabilitación de paredes de bahareque, pero se recomienda la mezcla de barro con cemento y bloques de arcilla por ser la que obtuvo los valores más bajos de absorción de agua, es decir, fue la más impermeable.

Los porcentajes de cemento y cal no fueron lo suficientemente críticos para considerarse perjudiciales desde el punto de vista de la sostenibilidad y tienen un impacto moderado en el ambiente. Además, se recicla material considerado desperdicio como los restos de bloques de cemento y de arcilla. El uso de estos escombros permite proponer estas mezclas bajo el marco de la sostenibilidad.

\section{Referencias}

Avgustinik A. Cerámica, traducido del ruso por DIORKI, traductores, Barcelona, Editorial Reverté SA, 1983, pp. 56.

Díaz J., Guinea M., Rohmer E., Salas J. Primeros resultados del trabajo de investigación sobre la tierra como material de construcción, en el IETcc. Informes de la Construcción, volumen 37 (número 377), 1986: 5 -21.

Craterre-Doat P., Hays A., Houben H., Matuk S., Vitoux F. Construir con tierra. Tomo II, Colombia, Fondo Rotatorio Editorial, 1990, pp. 132.

Henneberg-De León A. Paredes de bahareque en el Estado Zulia. Estudio integral para su rehabilitación sostenible, tesis (doctorado 
en arquitectura), Maracaibo, Venezuela, Universidad del Zulia, Maracaibo, Venezuela, 2010, 372 p.

Henneberg-De León A. Aproximación a un estudio sobre las lesiones del bahareque en el estado Zulia, Venezuela. Algunas recomendaciones para su intervención. Informes de la Construcción, volumen 64 (número 525), 2012: 63-74.

Minke G. Manual de construcción en tierra, 2a ed., Uruguay, Editorial Fin de Siglo, 2005, pp. 53-55.

Navarro N. Estabilización de suelos, en: I Curso Internacional Diseño y Construcción con Tierra (1er, 1995, Mérida, Venezuela), CYTED Red XIV.a- Habiterra, Programa Iberoamericano de Ciencia y Tecnología para el desarrollo, 1995, pp. 59-60.

Viñuales G.M. Restauración de arquitectura de tierra, Tucumán, Argentina, Editorial del Instituto Argentino de Investigaciones de Historia de la Arquitectura del Urbanismo, Universidad Nacional de Tucumán, 1981, p. 38.

\section{Este artículo se cita: \\ Citación estilo Chicago}

Henneberg-De León Andrea Mara, Daisy Briceño. Ensayos a mezclas de barro estabilizadas para el relleno y empañetado de paredes de bahareque. Ingeniería Investigación y Tecnología, XVII, 01 (2016): 143-154.

\section{Citación estilo ISO 690}

Henneberg-De León A.M., Briceño-D. Ensayos a mezclas de barro estabilizadas para el relleno y empañetado de paredes de bahareque. Ingeniería Investigación y Tecnología, volumen XVII (número 1), enero-marzo 2016: 143-154.

\section{Semblanzas de los autores}

Andrea Mara Henneberg-De León. Es arquitecta y profesora titular a dedicación exclusiva, adscrita al Departamento de Construcción y Tecnología en Arquitectura en la Facultad de Arquitectura y Diseño de la Universidad del Zulia, Maracaibo, Venezuela. Especialista en construcción en obras civiles y doctora en Arquitectura. Actualmente es presidenta del Consejo de Apelaciones de la Universidad del Zulia, directora del Departamento de Construcción y Tecnología en Arquitectura, miembro del comite editorial de Revista Portafolio. Sus líneas de investigación son: construcciones de bahareque, rehabilitación sostenible, patrimonio regional, recursos educacionales y herramientas automatizadas educativas.

Daisy Briceño. Es ingeniera civil y profesora asociada a medio tiempo adscrita al Departamento de Construcción y Tecnología en Arquitectura en la Facultad de Arquitectura y Diseño de la Universidad del Zulia, Maracaibo, Venezuela. Es especialista en construcción en obras civiles. Ejerce libremente su profesion en una firma de ingenieros consultores, con énfasis en cálculo y diseño estructural. Sus líneas de investigación son: ensayos de materiales, estructuras sostenibles, sistemas estructurales. 